PROCEEDINGS OF THE

AMERICAN MATHEMATICAL SOCIETY

Volume 130, Number 5, Pages 1285-1292

S 0002-9939(01)06190-1

Article electronically published on October 24, 2001

\title{
FLAT COVERS AND COTORSION ENVELOPES OF SHEAVES
}

\author{
EDGAR ENOCHS AND LUIS OYONARTE
}

(Communicated by Wolmer V. Vasconcelos)

\begin{abstract}
In this paper we prove that any sheaf of modules over any topological space (in fact, any $\mathcal{O}$-module where $\mathcal{O}$ is a sheaf of rings on the topological space) has a flat cover and a cotorsion envelope. This result is very useful, as we shall explain later in the introduction, in order to compute cohomology, due to the fact that the category of sheaves $(\mathcal{O}$-modules) does not have in general enough projectives.
\end{abstract}

\section{INTRODUCTION AND PRELIMINARIES}

In the category of $\mathcal{O}$-modules on a topological space $X$ (where $\mathcal{O}$ is a sheaf of rings on $X$ ) it is known that, in general, there are not enough projectives. However, every $\mathcal{O}$-module is the quotient of a flat $\mathcal{O}$-module and so every $\mathcal{O}$-module has a flat resolution. These flat resolutions can be used to compute homology (i.e. to compute $\operatorname{Tor}_{n}^{\mathcal{O}}(F, G)$ for right and left $\mathcal{O}$-modules $F$ and $G$ ). However, these flat resolutions are not adequate for computing cohomology. This means, for example, that when applying $\operatorname{Hom}(G,-)$ to two flat resolutions of some $F$, the two resulting complexes may fail to have the same cohomology groups. In this article we will show that every $\mathcal{O}$-module has a flat precover, and in fact that it has a flat cover (Theorem 2.7). Such a precover is necessarily epimorphic. Then, it is clear that any two flat resolutions of some $\mathcal{O}$-module which are computed using successive flat precovers are homotopically equivalent, and so both will give the same homology and cohomology groups when any additive functor (whether covariant or contravariant) is applied to them. In some sense this remedies the deficiency of not having enough projectives in the category of $\mathcal{O}$-modules

In order to prove the existence of these flat covers we will first prove their existence in the category of $\mathcal{O}$-premodules (i.e. where we have presheaves instead of sheaves) in Theorem 2.6] Then we will see that the flat cover of an $\mathcal{O}$-module in the category of $\mathcal{O}$-premodules is in fact an $\mathcal{O}$-module and so is a flat cover in the category of $\mathcal{O}$-modules.

We will also prove that every $\mathcal{O}$-module has a cotorsion envelope (Theorem 3.2).

If $\mathcal{F}$ is a class of objects in a category $\mathcal{C}$ and if $X$ is an object of $\mathcal{C}$, then an $\mathcal{F}$-precover of $X$ is a morphism $\varphi: F \rightarrow X$ where $F \in \mathcal{F}$ and such that $\operatorname{Hom}\left(F^{\prime}, F\right) \rightarrow \operatorname{Hom}\left(F^{\prime}, X\right)$ is surjective for all $F^{\prime} \in \mathcal{F}$. If, moreover, $\varphi \circ f=\varphi$ for

Received by the editors July 26, 2000 and, in revised form, November 3, 2000.

2000 Mathematics Subject Classification. Primary 16G10, 18F20; Secondary $18 \mathrm{E} 15$.

Key words and phrases. Flat (pre)cover, cotorsion (pre)envelope, (pre)sheaf, $\mathcal{O}$-(pre)module, Grothendieck category. 
$f: F \rightarrow F$ implies $f$ is an automorphism of $F$, then $\varphi: F \rightarrow X$ is called an $\mathcal{F}$-cover of $X$. If an $\mathcal{F}$-cover of $X$ exists, it is unique up to isomorphism. The dual notions are those of an $\mathcal{F}$-pre-envelope and envelope of $X$. Precovers, covers, pre-envelopes and envelopes are often named according to the class $\mathcal{F}$. So, for example, a flat cover in the category of left $R$-modules over some ring $R$ is an $\mathcal{F}$-cover where $\mathcal{F}$ is the class of flat left $R$-modules. See $[5$ for other examples.

If $\mathcal{A}$ is an abelian category and $\mathcal{F}$ is a class of objects of $\mathcal{A}$, then by $\mathcal{F}^{\perp}$ we mean the class of objects $C$ of $\mathcal{A}$ such that $\operatorname{Ext}^{1}(F, C)=0$ for all $F \in \mathcal{F}$. By ${ }^{\perp} \mathcal{F}$ we mean the class of $G$ such that $\operatorname{Ext}^{1}(G, F)=0$ for all $F \in \mathcal{F}$. A morphism $\varphi: F \rightarrow A$ in $\mathcal{A}$ where $F \in \mathcal{F}$, where $\varphi$ is an epimorphism, and such that $\operatorname{ker}(\varphi) \in \mathcal{F}^{\perp}$ is called a special $\mathcal{F}$-precover of $A$. A special precover is a precover since if $F^{\prime} \in \mathcal{F}$, we have the exact sequence

$$
\operatorname{Hom}\left(F^{\prime}, F\right) \rightarrow \operatorname{Hom}\left(F^{\prime}, A\right) \rightarrow \operatorname{Ext}^{1}\left(F^{\prime}, \operatorname{ker}(\varphi)\right)=0 .
$$

The dual notion is that of a special pre-envelope.

Given a class $\mathcal{F}$ of objects in an abelian category $\mathcal{A}$, we say that $\left(\mathcal{F}, \mathcal{F}^{\perp}\right)$ is cogenerated by a set if there exists a set $\mathcal{S} \subseteq \mathcal{F}$ (and so not just a class) such that $\mathcal{S}^{\perp}=\mathcal{F}^{\perp}$ (see Salce [11] for this terminology).

If $\mathcal{A}$ is an abelian category with small direct limits and if $X$ is an object of $\mathcal{A}$, we say that $X$ is the continuous union of the chain of subobjects $\left\{X_{\alpha} ; \alpha<\lambda\right\}$ ( $\lambda$ an ordinal number) of $X$ if $X_{\alpha} \subseteq X_{\beta}$ when $\alpha \leq \beta<\lambda$, if $X_{\beta}=\sum_{\alpha<\beta} X_{\alpha}$ where $\beta<\lambda$ is a limit ordinal, and if $X=\sum_{\alpha<\lambda} X_{\alpha}$.

Some of the results we will now show have not appeared in print and so we will give their full statements (but without proofs). Also, we will make some slight changes in the hypothesis. But in each case the proof will easily still go through.

Theorem 1.1 (Eklof and Trlifaj [4). Let $\mathcal{A}$ be a Grothendieck category. Let $\mathcal{F}$ be a class of objects of $\mathcal{F}$ such that if an object $F$ of $\mathcal{A}$ is the continuous union of a chain of subobjects $\left\{F_{\alpha} ; \alpha<\lambda\right\}$ with $F_{0} \in \mathcal{F}$ and $F_{\alpha+1} / F_{\alpha} \in \mathcal{F}$ when $\alpha+1<\lambda$, then, $F \in \mathcal{F}$. Suppose that $\left(\mathcal{F}, \mathcal{F}^{\perp}\right)$ is cogenerated by a set. Then, every object of $\mathcal{A}$ has a special $\mathcal{F}^{\perp}$-pre-envelope. Furthermore, if $\mathcal{A}$ has enough projectives and if these are all in $\mathcal{F}$, then every object has a special $\mathcal{F}$-precover. If $\mathcal{F}$ is also closed under direct summands, then ${ }^{\perp}\left(\mathcal{F}^{\perp}\right)=\mathcal{F}$ (so in the language of Salce [11], $\left(\mathcal{F}, \mathcal{F}^{\perp}\right.$ ) is a cotorsion theory on $\mathcal{A}$ ).

Proof. See [4, Theorem 2] (or 11 for a more categorical argument) for all but the last claim. For this let $G \in^{\perp}\left(\mathcal{F}^{\perp}\right)$. Then $G$ has a special $\mathcal{F}$-precover. So there is an exact sequence

$$
0 \rightarrow C \rightarrow F \rightarrow G \rightarrow 0
$$

with $C \in \mathcal{F}^{\perp}$ and $F \in \mathcal{F}$. Since $G \in^{\perp}\left(\mathcal{F}^{\perp}\right)$, this sequence splits and so $G$ is a direct summand of $F$. Hence $G \in \mathcal{F}$.

We note that Eklof and Trlifaj credit the idea for the proof of this result to [6]. Also see [10, Lemma 3.3] or [7, Theorem 2.1.14] for a similar argument.

We will also use:

Theorem 1.2. Let $\mathcal{A}$ be a Grothendieck category and $\mathcal{F}$ a class of objects of $\mathcal{A}$ which is closed under small direct limits. If an object $X$ of $\mathcal{A}$ has an $\mathcal{F}$-precover, then it has an $\mathcal{F}$-cover. 
Proof. This a straightforward modification of the "only if" part of 5. Theorem 2.1].

Now let $X$ be a topological space and let $\mathcal{O}$ be a sheaf of rings on $X$. We let $\mathcal{A}$ be the abelian category of $\mathcal{O}$-premodules (or in Serre's terminology [12], the category of presheaves of $\mathcal{O}$-modules). We will say that an $\mathcal{O}$-premodule $F$ is said to be flat if $\lim _{\rightarrow x \in U} F(U)=F_{x}$ (where the limit is taken over all open $U \subseteq X$ with $x \in U$ ) is a flat $\mathcal{O}_{x}$-module for all $x \in X$. This agrees with the usual definition of flat in the category of $\mathcal{O}$-modules but differs from Swan's [13 pg. 195]. It is convenient for our purposes since our interest is the category of $\mathcal{O}$-modules. A premodule which is flat using Swan's definition is also flat in our sense.

It is clear that if $F$ is a continuous union of a chain of subobjects $\left\{F_{\alpha} ; \alpha<\lambda\right\}$ with $F_{0}$ and each $F_{\alpha+1} / F_{\alpha}$ flat, then $F$ is flat. Also, any direct summand of a flat $\mathcal{O}$-premodule is flat, and if we denote by $\mathcal{F}$ the class of all flat $\mathcal{O}$-premodules, $\mathcal{F}$ is closed under small direct limits. Hence, to be able to apply Theorem 1.1 (and Theorem 1.2) with these $\mathcal{A}$ and $\mathcal{F}$ we only need to prove the easy result that $\mathcal{A}$ has enough projectives and then that $\left(\mathcal{F}, \mathcal{F}^{\perp}\right)$ is cogenerated by a set.

We will need one more reduction of our problem. This is provided by the following result.

Theorem 1.3 (Eklof [3]). Let $F$ be an object in a Grothendieck category. Let $F$ be the continuous union of a chain of subobjects $\left\{F_{\alpha} ; \alpha<\lambda\right\}$ and let $C$ be an object of $\mathcal{A}$. Then, if $\operatorname{Ext}^{1}\left(F_{0}, C\right)=0$ and $\operatorname{Ext}^{1}\left(F_{\alpha+1} / F_{\alpha}, C\right)=0$ when $\alpha+1<\lambda$, then $\operatorname{Ext}^{1}(F, C)=0$.

This result is useful in proving that $\left(\mathcal{F}, \mathcal{F}^{\perp}\right)$ is cogenerated by a set. It is used in this fashion. If $\mathcal{S} \subseteq \mathcal{F}$ is a set and if every $F \in \mathcal{F}$ is the union of a continuous chain $\left\{F_{\alpha} ; \alpha<\lambda\right\}$ of a subobject with $F_{0}$ and each $F_{\alpha+1} / F_{\alpha}$ isomorphic to an object of $\mathcal{S}$, then the theorem implies $\mathcal{S}^{\perp}=\mathcal{F}^{\perp}$ and so that $\left(\mathcal{F}, \mathcal{F}^{\perp}\right)$ is cogenerated by a set.

Suppose we are in a concrete category (so the objects are sets with some structure) satisfying the hypotheses of Theorem 1.3. Suppose that $\mathcal{F}$ is a class of objects that is closed under direct unions. If we can find a cardinal $\aleph$ such that for $x \in F \in \mathcal{F}$ there is a subobject $S \subseteq F$ with $x \in S$, with $|S| \leq \aleph$ and with $S, F / S \in \mathcal{F}$. Then it is easy to see that we can write $F$ as the union of a continuous chain $\left\{F_{\alpha} ; \alpha<\lambda\right\}$ of subobjects with $\left|F_{0}\right| \leq \aleph,\left|F_{\alpha+1} / F_{\alpha}\right| \leq \aleph($ for $\alpha+1<\lambda)$ and with $F_{0}, F_{\alpha+1} / F_{\alpha} \in \mathcal{F}$. Then if $\mathcal{S}$ is a set of representatives of the objects $S \in \mathcal{F}$ with $|S| \leq \aleph$, we see by Theorem 1.3 that $\mathcal{S}^{\perp}=\mathcal{F}^{\perp}$ and so that $\left(\mathcal{F}, \mathcal{F}^{\perp}\right)$ is cogenerated by a set.

In the next section we will prove that the category $\mathcal{A}$ of $\mathcal{O}$-premodules (with $\mathcal{O}$ a presheaf of rings on a topological space) has enough projectives and that with $\mathcal{F}$ the class of flat $\mathcal{O}$-premodules there exists a cardinal $\aleph$ as above.

\section{FLAT COVERS}

We let $\mathcal{O}$ be a sheaf of rings on the topological space $X$. Let $U$ be open and let $M$ be any left $\mathcal{O}(U)$-module. We define an $\mathcal{O}$-premodule $F_{U, M}$ by

$$
F_{U, M}(V)=\left\{\begin{array}{cl}
\mathcal{O}(V) \otimes_{\mathcal{O}(U)} M & \text { if } V \subseteq U, \\
0 & \text { if } V \nsubseteq U,
\end{array}\right.
$$


where if $W \subseteq V$ are open sets the restriction map $F_{U, M}(V) \rightarrow F_{U, M}(W)$ is 0 if $V \nsubseteq U$, and is the map $\mathcal{O}(V) \otimes_{\mathcal{O}(U)} M \rightarrow \mathcal{O}(W) \otimes_{\mathcal{O}(U)} M$ induced by the restriction $\mathcal{O}(V) \rightarrow \mathcal{O}(W)$ and by $i d_{M}$ if $V \subseteq U$. It is clear that $F_{U, M}$ is an $\mathcal{O}$-premodule.

Let $G$ be any $\mathcal{O}$-premodule and let $f: M \rightarrow G(U)$ be $\mathcal{O}(U)$-linear. It is immediate that there is a unique morphism $F_{U, M} \rightarrow G$ of $\mathcal{O}$-premodules with $F_{U, M}(U)=M \rightarrow G(U)$ being $f$. Using this fact and the fact that epimorphisms of $\mathcal{O}$-premodules $F \rightarrow G$ are those that $F(V) \rightarrow G(V)$ is surjective for all $V$, it is easy to see that $F_{U, M}$ is a projective $\mathcal{O}$-premodule if $M$ is a projective $\mathcal{O}(U)$-module (this is just the typical adjoint procedure for producing projectives).

Note that $\left(F_{U, M}\right)_{x}=0$ if $x \notin U$ and $\left(F_{U, M}\right)_{x}=\mathcal{O}_{x} \otimes_{\mathcal{O}(U)} M$ if $x \in U$. So $F_{U, M}$ is flat if $M$ is flat.

Also, we see that any $\mathcal{O}$-premodule $G$ can be written as the quotient of a direct sum of $F_{U, P}$ 's for open $U \subseteq X$ and projective $\mathcal{O}(U)$-modules $P$. Hence, we have proved the next result.

Proposition 2.1. For any sheaf of rings $\mathcal{O}$ on a topological space $X$, the category of $\mathcal{O}$-premodules has enough projectives.

We will now prove the other desired condition on the class of flat $\mathcal{O}$-premodules. We recall the following.

Lemma 2.2 (Jensen and Lenzing [9, Theorem 6.4]). If $R$ is a ring and $|R| \leq \aleph$ where $\aleph$ is an infinite cardinal number, and if $Y \subseteq M$ is a subset of a left $R$-module $M$ with $|Y| \leq \aleph$, then there exists a pure submodule $N \subseteq M$ such that $Y \subseteq N$ and $|N| \leq \aleph$.

Corollary 2.3. Every left $R$-module $M$ is the continuous union of a chain of pure submodules $\left\{M_{\alpha} ; \alpha<\lambda\right\}$ with $\left|M_{0}\right| \leq \aleph$ and $\left|M_{\alpha+1} / M_{\alpha}\right| \leq \aleph$ for $\alpha+1<\lambda$.

Proof. If we suppose that the elements of $M$ are well ordered, we construct the $M_{\alpha}$ transfinitely. Let $M_{0} \subseteq M$ be pure and contain the least element of $M$, with $\left|M_{0}\right| \leq \aleph$.

If $M_{\alpha}$ has been constructed, let $M_{\alpha+1} / M_{\alpha} \subseteq M / M_{\alpha}$ be pure where $M_{\alpha+1}$ contains the least element of $M$ not in $M_{\alpha}$ (of course, unless $M_{\alpha}=M$, in which case let $\lambda=\alpha+1$ ) and with $\left|M_{\alpha+1} / M_{\alpha}\right| \leq \aleph$. For every limit ordinal $\beta<\lambda$ let $M_{\beta}=\bigcup_{\alpha<\beta} M_{\alpha}$ when $M_{\alpha}$ has been constructed for all $\alpha<\beta$.

We want to prove an analogous result for the category of $\mathcal{O}$-premodules. Given an $\mathcal{O}$-premodule $F$, we let $|F|=|\coprod F(U)|$ with the coproduct taken over all open $U \subseteq X$.

Given an $\mathcal{O}$-premodule $F$, we will say that a subpremodule $G \subseteq F$ is pure if $G(U)$ is pure in $F(U)$ (as $\mathcal{O}(U)$-modules) for all open $U \subseteq X$.

Proposition 2.4. For the sheaf of rings $\mathcal{O}$ on $X$, let $|\mathcal{O}| \leq \aleph$ where $\aleph$ is an infinite cardinal number. Let $F$ be an $\mathcal{O}$-premodule and let $Y \subseteq F\left(U^{\prime}\right)$ be a subset for some open $U^{\prime} \subseteq X$ with $|Y| \leq \aleph$. Then, there exists a pure subpremodule $G \subseteq F$ with $Y \subseteq G\left(U^{\prime}\right)$ and $|G| \leq \aleph$.

Proof. We first make some observations. For any open $U,|\mathcal{O}(U)| \leq \aleph$ and $|\mathcal{U}| \leq \aleph$ where $\mathcal{U}$ is the set of all open subsets $U \subseteq X$. If $Z \subseteq \bigcup_{U \in \mathcal{U}} F(U)$ is a subset, then the subpremodule $H$ of any premodule $F$ generated by $Z$ is such that $H(U)=$ $\sum_{s \in Z \cap F(U)} \mathcal{O}(U) \cdot s$. So if $|Z| \leq \aleph$, then $|H| \leq \aleph$. 
Let $\lambda$ be the first limit ordinal such that $|\mathcal{U}| \leq|\lambda| \leq \aleph$ and suppose that $\left\{U_{\alpha}\right.$; $\alpha<\lambda\}$ is the family of all open $U \subseteq X$ (with possible repetition in case $|\mathcal{U}|<|\lambda|$ ) with $U_{0}=U^{\prime}$.

Using transfinite induction we construct an increasing sequence $\left\{G_{\alpha} ; \alpha<\lambda\right\}$ of subpremodules of $F$. By Lemma 2.2, we have $Y \subseteq N \subseteq F\left(U^{\prime}\right)$ with $N$ a pure $\mathcal{O}\left(U^{\prime}\right)$ submodule of $F\left(U^{\prime}\right)$ and with $|N| \leq \aleph$. Let $G_{0} \subseteq F$ be the subpremodule generated by $N$. Then $Y \subseteq G_{0}\left(U_{0}\right)=N$ (so $G_{0}\left(U_{0}\right) \subseteq F\left(U_{0}\right)$ is pure) and $\left|G_{0}\right| \leq \aleph$. We have $G_{0}\left(U_{1}\right) \subseteq F\left(U_{1}\right)$ and $\left|G_{0}\left(U_{1}\right)\right| \leq \aleph$ so there is a pure submodule $N_{1} \subseteq F\left(U_{1}\right)$ with $G_{0}\left(U_{1}\right) \subseteq N_{1} \subseteq F\left(U_{1}\right)$ and $\left|N_{1}\right| \leq \aleph$. Let $G_{1} \subseteq F$ be the subpremodule generated by $N_{1} \cup\left(\bigcup_{U \in \mathcal{U}} G_{0}(U)\right)$. Then $G_{1}\left(U_{1}\right)=N_{1}$ and $\left|G_{1}\right| \leq \aleph$.

So, in general, having constructed $G_{\alpha}$ with $\alpha+1<\lambda$ and $\left|G_{\alpha}\right| \leq \aleph$, we let $G_{\alpha+1}$ be generated by $N_{\alpha+1} \cup\left(\bigcup_{U \in \mathcal{U}} G_{\alpha}(U)\right)$ where $G_{\alpha}\left(U_{\alpha+1}\right) \subseteq N_{\alpha+1} \subseteq F\left(U_{\alpha+1}\right)$, $N_{\alpha+1} \subseteq F\left(U_{\alpha+1}\right)$ pure and $\left|N_{\alpha+1}\right| \leq \aleph$. So we get that $\left|G_{\alpha+1}\right| \leq \aleph$ and that $G_{\alpha+1}\left(U_{\alpha+1}\right) \subseteq F$ pure.

If $\beta<\lambda$ is a limit ordinal, we first let $G_{\beta}^{\prime}=\bigcup_{\alpha<\beta} G_{\alpha}$ (getting $\left|G_{\beta}^{\prime}\right| \leq \aleph$ ). Then find a pure submodule $N_{\beta} \subseteq F\left(U_{\beta}\right)$ with $G_{\beta}^{\prime}\left(U_{\beta}\right) \subseteq N_{\beta} \subseteq F\left(U_{\beta}\right)$ and with $\left|N_{\beta}\right| \leq \aleph$, and let $G_{\beta}$ be the subpremodule of $F$ generated by $N_{\beta} \cup\left(\bigcup_{U \in \mathcal{U}} G_{\beta}^{\prime}(U)\right)$.

So we get an increasing sequence $\left\{G_{\alpha} ; \alpha<\lambda\right\}$ of subpremodules of $F$ with $\left|G_{\alpha}\right| \leq \aleph$ for all $\alpha<\lambda$, with $G_{\alpha}\left(U_{\alpha}\right) \subseteq F\left(U_{\alpha}\right)$ pure for all $\alpha<\lambda$ and with $Y \subseteq G_{0}\left(U_{0}\right)$.

Letting $G_{\lambda}^{\prime}=\bigcup_{\alpha<\lambda} G_{\alpha}$ we have $\left|G_{\lambda}^{\prime}\right| \leq \aleph$. Now using the same procedure as above we can find a subpremodule $G_{\lambda} \subseteq F$ with $G_{\lambda}^{\prime} \subseteq G_{\lambda}$, with $\left|G_{\lambda}\right| \leq \aleph$ and with $G_{\lambda}\left(U_{0}\right) \subseteq F\left(U_{0}\right)$ pure. Then we find $G_{\lambda} \subseteq G_{\lambda+1} \subseteq F$ with $\left|G_{\lambda+1}\right| \leq \aleph$ and $G_{\lambda+1}\left(U_{1}\right) \subseteq F\left(U_{1}\right)$ pure. So we can construct the increasing sequence $\left\{G_{\lambda+\alpha} ; \alpha<\right.$ $\lambda\}$ with $\left|G_{\lambda+\alpha}\right| \leq \aleph$ and $G_{\lambda+\alpha}\left(U_{\alpha}\right) \subseteq F\left(U_{\alpha}\right)$ pure for every $\alpha$.

After this sequence has been constructed, we can construct the increasing sequence $\left\{G_{2 \lambda+\alpha} ; \alpha<\lambda\right\}$ in a similar manner, and so finally we have the increasing sequences

$$
\left\{G_{n \lambda+\alpha} ; \alpha<\lambda\right\} \quad n \geq 0
$$

with $\left|G_{n \lambda+\alpha}\right| \leq \aleph$ for each $n$ and $\alpha$ and with $G_{n \lambda+\alpha}\left(U_{\alpha}\right) \subseteq F\left(U_{\alpha}\right)$ pure for each $n$ and $\alpha$.

We let $G=\sup _{n, \alpha} G_{n \lambda+\alpha}$. Then $|G| \leq \aleph$. Also, we see that for any $\alpha<\lambda$, $G\left(U_{\alpha}\right)=\bigcup_{n=0}^{\infty} G_{n \lambda+\alpha}\left(U_{\alpha}\right)$. But $G_{n \lambda+\alpha}\left(U_{\alpha}\right) \subseteq F\left(U_{\alpha}\right)$ is pure for each $n \geq 0$, so $G\left(U_{\alpha}\right) \subseteq F\left(U_{\alpha}\right)$ is pure.

Since $Y \subseteq G_{0}\left(U_{0}\right) \subseteq G\left(U_{0}\right)=G\left(U^{\prime}\right)$, we see that we have the desired $G$.

Corollary 2.5. Any $\mathcal{O}$-premodule $F$ is the union of a continuous increasing sequence of pure subpremodules $\left\{F_{\alpha} ; \alpha<\lambda\right\}$ with $\left|F_{0}\right| \leq \aleph$ and with $\left|F_{\alpha+1} / F_{\alpha}\right| \leq \aleph$ whenever $\alpha+1<\lambda$.

Proof. This is proved in the same way that Corollary 2.3 was proved.

It is known that the categories of $\mathcal{O}$-(pre)modules have enough flat objects, that is, every $\mathcal{O}$-(pre)module is a quotient of a flat $\mathcal{O}$-(pre)module (see for example [13. Corollary 6.8]). In fact, given two flat resolutions of an $\mathcal{O}$-module $A$, and two morphisms $f, g$ between these resolutions, over the same morphism $\left(i d_{A}: A \rightarrow A\right)$, one can find a third flat resolution of $A$, and a morphism of resolutions $h$ over $i d_{A}$, in such a way that $f h$ and $g h$ are homotopic ([13, Lemma 4.2]). However, one cannot still assert that two arbitrary flat resolutions of $A$ are homotopically 
equivalent. This problem will be solved when one can assure the existence of flat precovers, since then one can construct flat resolutions of an $\mathcal{O}$-(pre)module by taking successive flat precovers. It is then clear that all flat resolutions of an $\mathcal{O}$ (pre)module constructed in such a way are homotopically equivalent (so all of them have the same homology and cohomology groups no matter what additive functor is applied to them). The next two results will be devoted to prove the existence of not just flat precovers but flat covers of $\mathcal{O}$-premodules and $\mathcal{O}$-modules. First, though, we emphasize the distinction between a flat cover in the category of premodules and in the category of modules. In the first case the $F$ in question is only required to be a premodule (but a flat one) and in the second case is required to be a module.

Theorem 2.6. If $\mathcal{O}$ is a sheaf of rings on the topological space $X$, then every $\mathcal{O}$-premodule has a flat cover.

Proof. We let $\mathcal{F}$ be the class of flat $\mathcal{O}$-premodules and let $F \in \mathcal{F}$. By Corollary 2.5 we have that $F$ is the union of a continuous increasing sequence $\left\{F_{\alpha} ; \alpha<\lambda\right\}$ of pure subpremodules. Since $F_{\alpha}(U) \subseteq F(U)$ is pure for all $U,\left(F_{\alpha}\right)_{x} \subseteq F_{x}$ is pure for all $x \in X$. Thus, $\left(F_{\alpha}\right)_{x}$ is flat for all $x \in X$ and all $\alpha<\lambda$, and then, for $\alpha+1<\lambda$, we similarly get that $\left(F_{\alpha}\right)_{x} \subseteq\left(F_{\alpha+1}\right)_{x}$ is pure. Therefore, $\left(F_{\alpha+1} / F_{\alpha}\right)_{x} \cong$ $\left(F_{\alpha+1}\right)_{x} /\left(F_{\alpha}\right)_{x}$ is flat for all $x \in X$, so $F_{\alpha+1} / F_{\alpha}$ is a flat premodule for all $\alpha+1<\lambda$.

Similarly, $F_{0}$ is flat, so by the remarks at the end of Section 1 we see that $\left(\mathcal{F}, \mathcal{F}^{\perp}\right)$ is cogenerated by a set. Then, by Theorems 1.1 and 1.2 (with $\mathcal{A}$ the category of $\mathcal{O}$-premodules) we get the result.

We now prove our main result.

Theorem 2.7. If $\mathcal{O}$ is a sheaf of rings on a topological space, then every $\mathcal{O}$-module has a flat cover (in the category of $\mathcal{O}$-modules). In fact, if $F \rightarrow G$ is a flat cover of the $\mathcal{O}$-module $G$ in the category of $\mathcal{O}$-premodules, then $F$ is an $\mathcal{O}$-module and $F \rightarrow G$ is a flat cover in the category of $\mathcal{O}$-modules.

Proof. We recall the process of sheafification of a presheaf, or in other words, the way to create an $\mathcal{O}$-module from an $\mathcal{O}$-premodule (see for example [ $\underline{8}$, Section II.2]). Given the premodule $F$, there is a morphism $F \rightarrow \bar{F}$ of premodules with $\bar{F}$ an $\mathcal{O}$ module such that every morphism $F \rightarrow G$ of $F$ into an $\mathcal{O}$-module $G$ can be factored uniquely $F \rightarrow \bar{F} \rightarrow G$. Also, by the construction of $\bar{F}, F_{x}=\bar{F}_{x}$ for all $x \in X$, so $F$ is a flat $\mathcal{O}$-premodule if and only if $\bar{F}$ is a flat $\mathcal{O}$-module (this can also be seen in [13, Lemma 6.7]).

Now let $G$ be an $\mathcal{O}$-module. By Theorem 2.6, $G$ has a flat cover $F \rightarrow G$ in the category of premodules, but then we have the factorization $F \rightarrow \bar{F} \rightarrow G$. Since $\bar{F}$ is flat (as a premodule), $\bar{F} \rightarrow G$ can be factored $\bar{F} \rightarrow F \rightarrow G$. But since $F \rightarrow G$ is a cover, $F \rightarrow \bar{F} \rightarrow F$ is an automorphism of $F$, so $F$ is a direct summand of the $\mathcal{O}$-module $\bar{F}$ and so is an $\mathcal{O}$-module. This means $F=\bar{F}$, so $F \rightarrow G$ is a flat cover in the category of $\mathcal{O}$-modules.

We note that Theorem 2.7 was proved for modules (when $X$ is a point) in [2].

\section{Cotorsion envelopes}

The next result is useful in proving the existence of envelopes.

Theorem 3.1. Let $\mathcal{A}$ be a Grothendieck category. Let $\mathcal{C}$ be a class of objects of $\mathcal{A}$ such that ${ }^{\perp} \mathcal{C}$ is closed under small direct limits. Then, if an object of $\mathcal{A}$ has a special $\left({ }^{\perp} \mathcal{C}\right)^{\perp}$-pre-envelope, it has a $\left({ }^{\perp} \mathcal{C}\right)^{\perp}$-envelope. 
Proof. This is essentially [14, Theorem 2.2.2]. The terminology is different and the proof given in [14] is for modules, but these are easily adjusted to this situation.

We let $\mathcal{O}$ be a sheaf of rings on $X$ and $\mathcal{A}$ the category of $\mathcal{O}$-modules. Let $\mathcal{F}$ be the class of flat $\mathcal{O}$-modules. Then, the objects $C \in \mathcal{F}^{\perp}$ are called cotorsion modules.

We want to appeal to the first part of Theorem 1.1 to guarantee that every object of $\mathcal{A}$ has a special $\mathcal{F}^{\perp}=\mathcal{C}$-pre-envelope. To do this we only need to argue that $\left(\mathcal{F}, \mathcal{F}^{\perp}\right)=(\mathcal{F}, \mathcal{C})$ is cogenerated by a set.

Given $F \in \mathcal{F}$, we appeal to Corollary 2.5 Hence, $F$ is the union of a continuous chain $\left\{F_{\alpha} ; \alpha<\lambda\right\}$ of pure subpremodules. Since $F_{\alpha} \subseteq F$ and $F$ is an $\mathcal{O}$-module, we get that $\bar{F}_{\alpha} \subseteq F$ (where $\bar{F}_{\alpha}$ is the sheafification of $F_{\alpha}$ ) and so $F$ is the union of the continuous chain $\left\{\bar{F}_{\alpha} ; \alpha<\lambda\right\}$ (in $\mathcal{A}$ ). Since $F_{0}$ and $F_{\alpha+1} / F_{\alpha}$ are flat presheaves (for $\alpha+1<\lambda$ ) we get that $\bar{F}_{0}$ and $\bar{F}_{\alpha+1} / \bar{F}_{\alpha}$ are flat sheaves, and since $\left|F_{0}\right| \leq \aleph$ and $\left|F_{\alpha+1} / F_{\alpha}\right| \leq \aleph$ (with the $\aleph$ of Corollary 2.5), we also get that $\left|\bar{F}_{0}\right| \leq \aleph^{\aleph}$, $\left|\bar{F}_{\alpha+1} / \bar{F}_{\alpha}\right| \leq \aleph^{\aleph}$ (because $|\mathcal{U}| \leq \aleph$, where $\mathcal{U}$ is the set of all open subsets of $X$ ). So now, arguing as in the proof of Theorem 2.6 we see that $(\mathcal{F}, \mathcal{C})$ is cogenerated by a set. Thus, Theorem 1.1 guarantees that every $\mathcal{O}$-module has a special $\mathcal{C}$-preenvelope. Then, by Theorem 3.1 (taking note that $\left({ }^{\perp} \mathcal{C}\right)^{\perp}=\left({ }^{\perp}\left(\mathcal{F}^{\perp}\right)\right)^{\perp}=\mathcal{F}^{\perp}=\mathcal{C}$ ), every $\mathcal{O}$-module has a $\mathcal{C}$-envelope, i.e. a cotorsion envelope.

Therefore, we have proved the next result.

Theorem 3.2. If $\mathcal{O}$ is a sheaf of rings on a topological space $X$, then every $\mathcal{O}$ module has a cotorsion envelope (in the category of $\mathcal{O}$-modules).

It would be interesting to know whether a modification of these methods proves the existence of flat covers in the category of quasi-coherent modules when $\mathcal{O}$ is the structure sheaf of a scheme.

\section{ACKNOWLEDGMENT}

The last author was supported by a grant from the Spanish Secretaría de Estado de Educación, Universidades, Investigación y Desarrollo. Subprograma General de Perfeccionamiento de Doctores en el Extranjero.

\section{REFERENCES}

[1] S.T. Aldrich, E. Enochs, J.R. García Rozas and L. Oyonarte, Covers and envelopes in Grothendieck categories. Flat covers of complexes with applications, Submitted.

[2] L. Bican, R. El Bashir and E. Enochs, All modules have flat covers, To appear in Bull. London Math. Soc.

[3] P.C. Eklof, Homological algebra and set theory, Trans. Amer. Math. Soc. 227 (1977), 207225. MR 56:11782

[4] P. Eklof and J. Trlifaj, How to make Ext vanish, Bull. London Math. Soc. 33 (2001), no. 1, 44-51. CMP 2001:05

[5] E. Enochs, Injective and flat covers, envelopes and resolvents, Israel J. Math. 39 (1981), 189-209. MR 83a:16031

[6] R. Göbel and S. Shelah, Cotorsion theories and splitters, Trans. Amer. Math. Soc. 352(11) (2000), 5357-5379. MR 2001b:20098

[7] M. Hovey, Model categories, Mathematical Surveys and Monographs, Vol. 63, American Mathematical Society, 1999. MR 99h:55031

[8] B. Iversen, Cohomology of sheaves, Springer-Verlag, 1986. MR 87m:14013

[9] C. Jensen and H. Lenzing, Model-theoretic algebra with particular emphasis on fields, rings, modules, Algebra, Logic and Applications, Vol. 2, Gordon and Breach, 1989. MR 91m:03038 
[10] D.G. Quillen, Homotopical algebra, Lecture Notes in Mathematics, Vol. 43, Springer-Verlag, 1967. MR 36:6480

[11] L. Salce, Cotorsion theories for abelian groups, in:Symposia Mathematica (Conference of abelian groups and their relationship to the theory of modules, INDAM, Rome, 1977) Vol. XXIII, Academic Press, London-New York, 1979, pp. 11-32. MR 81j:20078

[12] J.P. Serre, Faisceaux algébriques cohérents, Ann. Math. 61(2) (1955), 197-278. MR 16:953c

[13] R.S. Swan, Cup products in sheaf cohomology, pure injectives, and a substitute for projective resolutions, J. Pure Appl. Algebra 144 (1999), 169-211. MR 2001i:18016

[14] J. Xu, Flat covers of modules, Lecture Notes in Mathematics, Vol. 1634, Springer-Verlag, 1996. MR 98b:16003

Department of Mathematics, University of Kentucky, Lexington, Kentucky 405060027

E-mail address: enochs@ms.uky.edu

Departamento de Álgebra y Análisis Matemático, Universidad de Almería, 04071 Almería, SPAin

E-mail address: oyonarte@ual.es 